\title{
ESTIMATING SOIL/SEDIMENT PARTITION COEFFICIENTS FOR ORGANIC COMPOUNDS BY HIGH PERFORMANCE REVERSE PHASE LIQUID CHROMATOGRAPHY
}

\author{
Yu-Ping Chin ${ }^{1}$, Carole Sue Peven ${ }^{2}$ and Walter J. Weber JR \\ 'University of Michigan Environmental Engineering and Water Resources Program, Ann Arbor, \\ MI 48109 and ${ }^{2}$ Battelle New England Marine Research Laboratory, Duxbury, Mass., U.S.A.
}

(Received May 1987; accepted in revised form January 1988)

\begin{abstract}
High performance reverse phase liquid chromatography (HPRPLC) retention parameters were correlated to organic-carbon-normalized partition coefficient $\left(K_{\mathrm{oc}}\right)$ values for sorption of various uncharged organic substances onto sediments and soils in an attempt to devise a model for predicting sorption behavior. The results reveal a relationship between the logarithm of HPRPLC retention time and $\log K_{\text {c }}$ that appears to provide a reasonable means for estimating partitioning properties from HPRPLC data. The technique was compared to methods which utilize octanol/water partition coefficients $\left(K_{\text {ow }}\right)$ and aqueous solubilities as surrogate predictors of sorption behavior and was found to yield superior correlations in all cases tested.
\end{abstract}

Key words-sorption, partitioning, sediment, soil, reverse phase liquid chromatography, aqueous solubility, octanol/water partition coefficient

\section{INTRODUCTION}

Sorption phenomena exert important influences on diagenetic processes and the environmental fate of biogenic and anthropogenic organic substances in aqueous systems. Through sorption/desorption and sedimentation/resuspension phenomena natural soils and sediments can act as both aquatic sources and sinks for a host of organic compounds. The extent to which a particular organic species undergoes partitioning between sediments/soils and the aqueous phase is significantly affected by the physicochemical properties of that species. This suggests the possibility of developing means for estimation of the specific sorptive properties of particular organic substances from known properties and characteristic parameters, a highly desirable objective in terms of facilitating environmental fate and transport projections.

The sorption of uncharged organic species onto sediments and soils frequently has been observed to be linearly or quasi-linearly dependent upon solution phase concentration, particularly at very low concentration levels, and usually dependent upon the organic carbon content of the solid (Karickhoff, 1981, 1984; Chiou et al., 1983; Gschwend and $W u$, 1985; Brownawell, 1986; Brownawell and Farrington, 1986). This behavior is not unexpected, given the relatively low aqueous solubilities of such substances and their greater affinity for the organic phases associated with most natural solids. Many "state-ofthe-art" modeling procedures for predicting sorption phenomena in geochemical and environmental systems are predicated upon either the octanol/water partition coefficient $\left(K_{\text {ow }}\right)$ or the aqueous solubility $\left(S_{i}^{w}\right)$ of sorbing species (Karickhoff et al., 1979; Karickhoff, 1981, 1984; Chiou et al., 1979, 1983; Means et al., 1980, 1982; Brown and Flagg, 1981; Voice et al., 1983). Observed correlations between these two physicochemical parameters are attributable largely to their mutual dependence on the aqueous activity coefficient of a solute (Chiou et al., 1979, 1982, 1983; Miller et al., 1985). This has resulted in an extensive compilation of both $K_{\mathrm{ow}}$ (Leo and Hansch, 1979) and $S_{i}^{w}$ values (Yalkowsky and Valvani, 1980; Yalkowsky et al., 1983), both of which can be either determined experimentally (Haque and Schmedding, 1975; Karickhoff and Brown, 1979; Sutton and Calder, 1974; Miller et al. 1985) or estimated from empirical models based on additive free energy concepts (AFECs) (Fujita et al., 1964; Rekker, 1977; Arbuckle, 1983).

Efforts to model the sorption of hydrophobic nonelectrolytic organic substances using octanol/water partition coefficients or aqueous solubilities have proven reasonably successful (Karickhoff, 1981), but are still problematic. With respect to the octanol/water partition coefficient approach, Chiou et al. (1982) observed that the mutual saturation of octanol and water may alter the activity coefficient of an organic solute in each phase, particularly for very hydrophobic compounds. Voice and Weber (1985) suggested that $K_{\text {ow }}$ values may tend to underpredict the partitioning of hydrophobic compounds between 
water and particulate organic carbon because of mutual saturation effects. Moreover, experimental determinations of $K_{\text {ow }}$ are time consuming, and may lack analytical precision and accuracy, while estimates of these surrogate parameters derived from AFEC models cannot take into account steric effects or special molecular configurations.

Predictions of sorptive processes using aqueous solubility are even less satisfactory than those using octanol/water partition coefficients (Karickhoff et al., 1979). The predictive capabilities of aqueous solubility sorption models are greatly enhanced of one takes into account the crystal energy contribution of solutes that are solids at room temperature (Tsonopoulos and Prausnitz, 1971; Karickhoff, 1981; Weber et al., 1986; Yalkowsky and Valvani, 1980). Nonetheless, the application of aqueous solubility as a "predictor" of sorption offers no real advantage over the octanol/water partition coefficient approach, and experimental determinations can be exceedingly difficult to perform and reproduce.

High performance liquid chromatography has been utilized in organic geochemical and environmental applications to study the diagenesis of polynuclear hydrocarbons in lacustrine sediments (Wakeham et al., 1979), and quantify and separate complex mixtures of pollutants in water (Sorrell et al., 1977). In addition, high performance reverse phase liquid chromatography (HPRPLC) solute retention data has been utilized to rapidly estimate octanol/water partition coefficients (McCall, 1975; Vieth et al., 1979; Eadsforth and Moser, 1983; D'amboise and Hanai, 1982; McDuffie, 1980; Rapaport and Eisenreich, 1984; Burkhard et al., 1985; Chin et al., 1986) and aqueous solubilities (Hakfenschied and Tomlinson, 1981; Chin et al., 1986). Predictive modeling of the sediment/soil sorption behavior of uncharged organic solutes would seem a logical extension of this technique, in that the analysis would potentially be fast, accurate, and not require the use of such information as solute melting points and entropies of fusion. Rapaport and Eisenreich (1984) have in fact suggested that the partitioning behavior of an organic solute between the reverse phase, stationary phase and the mobile phase in a HPRPLC system is analogous to that involved in soil/sediment sorptive processes, and is therefore potentially a more accurate predictor of sorption than either aqueous solubility or octanol/water partition coefficients. This method, coupled with other qualitative techniques (e.g. mass spectrometry) might be particularly attractive for rapidly identifying and assessing the sorptive properties of diagenetically altered organic pollutants in marine and freshwater sediments (Burkhard $e t$ al., 1985). This paper investigates the feasibility of applying HPRPLC as a technique for estimating sediment partition coefficients, and compares the results with those obtained using octanol/water and aqueous solubility sorption models.

\section{THEORETICAL}

The distribution of a hydrophobic organic compound between water and a natural solid can be quantified through the use of several models. Weber et al. (1983) found that the Freundlich equation is particularly useful for analyzing sorption equilibrium data over large concentration ranges

$$
q_{\mathrm{c}}=K_{\mathrm{F}} C_{\mathrm{e}}^{n}
$$

where $q_{\mathrm{c}}$ and $C_{\mathrm{e}}$ are the concentrations of the sorbate in the solid and aqueous phases, and $K_{\mathrm{F}}$ and $n$ are empirical coefficients relating to the adsorption capacity and energy or intensity of adsorption respectively. If the value $n$ approaches unity, sorption can be quantified using a simple linear model

$$
K_{\mathrm{p}}=\frac{q_{\mathrm{e}}}{C_{\mathrm{e}}}
$$

where $K_{\mathrm{p}}$ is the equilibrium partition coefficient. It should be emphasized that equation (2) applies rigorously only to true absorption partitioning processes, or to adsorption phenomena in systems in which the equilibrium solution phase concentration is exceedingly low for any given solute (generally $<100 \mu \mathrm{g} \mathrm{I}^{-1}$ ); i.e. the "Henry's" sorption domain (Weber et al., 1983). Several investigators (Lambert et al., 1965; Karickhoff et al., 1979; Means et al., 1980; Brownawell and Farrington, 1986) observed that the sorption of uncharged hydrophobic organic compounds can be correlated to the organic carbon content of the sorbent. For such cases partition coefficient values for a variety of soils and sediments can be normalized in terms of the fraction of organic carbon associated with the sorbent to yield an organic-carbon-partition coefficient, $K_{\mathrm{oc}}$,

$$
K_{\mathrm{oc}}=K_{\mathrm{p}} / f_{\mathrm{oc}}
$$

Karickhoff et al. (1979) observed a generally good relationship between $K_{\mathrm{p}}$ and $K_{\mathrm{oc}}$, but noted deviations for sandy soils having low organic carbon content $\left(f_{\mathrm{oc}}<0.001\right)$. The $K_{\mathrm{oc}}$ values in the latter cases were lower by approximately a factor of two than those determined for high organic content silty sediments. Miller and Weber (1986) found a similar effect for the relative sorption of lindane onto a moderately high organic carbon soil and a sandy low organic carbon aquifer material. Equation (3) thus seems most appropriate for sediments and soils of moderately high organic carbon content.

The carbon normalized partition coefficient can be expressed in terms of the respective aqueous and organic carbon phase activity coefficients for a target compound $\gamma_{i}^{\mathrm{w}}$, and $\gamma_{i}^{\circ}$, and the respective molar volumes of the water and organic matter phases, $\nabla_{\mathrm{w}}$ and $\nabla_{0}$, (Chiou et al., 1983; Karickhoff, 1984) as

$$
K_{\mathrm{cc}}=\frac{\gamma_{i}^{\mathrm{w}}}{\eta_{i}^{\circ}}\left[\frac{\nabla_{\mathrm{w}}}{\nabla_{0}}\right]
$$

or, in logarithmic form,

$$
\log \left(K_{\mathrm{oc}}\right)=\log \left(\gamma_{i}{ }^{w}\right)+A
$$

where $A=\log \left(\nabla_{w} / \nabla_{0}\right)-\log \left(\gamma_{i}^{0}\right)$. The values $D_{0}$ and $\gamma_{i}^{\circ}$ cannot be reliably determined a priori, and must therefore be evaluated empirically.

Weber $e t$ al. (1986) demonstrated that the elution order of uncharged organic solutes in HPRPLC systems can be correlated to their mobile phase activity coefficients. They also observed that the logarithm of the activity coefficient of a target substance varied linearly with the amount of organic modifier present in the mobile phase, and were able to derive a correlation between aqueous phase solute activity coefficients and retention times of uncharged hydrophobic compounds

$$
\log \left(\gamma_{i}^{*}\right)=\alpha \log \left(t_{\mathrm{c}}\right)+\beta
$$

where $\alpha$ and $\beta$ are empirically determined constants, and $t_{c}$ is the solute retention time corrected to the retention 
time of an internal standard. Chin et al. (1986) observed an excellent correlation between the aqueous activity coefficients of several uncharged aromatic compounds and their respective HPRPLC retention times.

Combination of equations (5) and (6) yields

$$
\log \left(K_{\mathrm{oc}}\right)=\alpha \log \left(t_{\mathrm{c}}\right)+C
$$

where $C=A+\beta$. Equation (4) assumes that the aqueous activity coefficient exerts primary control on sorptive processes that can be directly determined with HPRPLC retention data and equation (6). Karickhoff (1981, 1984) also suggested that sorption is largely controlled by the aqueous phase solute activity coefficient. In addition, the partitioning behavior of organic compounds between a polar mobile phase and reverse phase packings is analogous to soil/sediment sorption phenomena. This paper examines the validity of the assumptions used to derive the above equations, and compares the suitability of the HPRPLC $-K_{o c}$ equation as a predictor of sorption behavior with the suitability of sorption models based on aqueous solubility and octanol/water partition coefficients.

\section{MATERIALS AND METHODS}

\section{Soil and rediment isotherms}

The sedisuent used for this study was obtained from Lake Michigan, offshore from South Haven, Mich. It was wet-sieved to $<\sigma 0 \mu \mathrm{m}$, freeze-dried, and stored in a sealed container at $-20^{\circ} \mathrm{C}$. The soil was collected from Michawye, Mich. It has a sandy character with a mean grain dia $0.432 \mathrm{~mm}$. This particular soil, chosen for its low and uniform organic carbon content, closely resembles sands in many aquifers, and thus provides a useful basis for measurement of sorption of organic compounds in subsurface systems. The sediment and soil were analyzed for organic carbon content using a persulfate oxidation technique, which yielded an organic content of $1.42 \%$ for the sediment and $0.13 \%$ for the soil.

The compounds included both aromatic and aliphatic chlorinated hydrocarbons; specifically, carbon tetrachloride (Kodak Co.), cis-chlordane (EPA, Research Triangle, N.C.), 1,2,4-trichlorobenzene (Aldrich Chemicals), 4,4-PCB, 2,5,2'PCB and 2,2, 4,5-PCB (Analabs/Foxboro Corp.). These compounds were chosen in part because their relative stability in the environment allows then to be used as tracers of environmental and geochemical fate and transport processes (Bopp et al., 1982; Wakeham and Farrington, 1980; Schwarzenbach and Westall, 1981; Brownawell, 1986; Brownawell and Farrington, 1986), in part because their toxicity poses as an environmental hazard, and in part because they are also relatively easy to assay.

Sorption equilibrium isotherm experiments using the offshore South Haven (OSH) lacustrine sediments and the nonvolatile organic compounds (PCBs, chlordane) were carried out using $150 \mathrm{ml}-\mathrm{Hypo}$-Vials (C.P. Pierce Co.) as individual completely mixed batch reactors (CMBRs) in so-called "bottle-point" experiments. In this type of measurement each point on the adsorption isotherm is determined in an individual CMBR by equilibrating a given solution of solute with a given quantity of sorbent. A $100 \mathrm{ml}$ aliquot of deionized distilled water was added to each vial and the target compound introduced to this water by direct injection in an acetone carrier to give solute concentrations ranging from 0 (blank) to approx. $50 \mu \mathrm{g} \mathrm{l}^{-1}$ (or $100 \mathrm{ug} \mathrm{1^{-1 }}$ in the case of 1,2,4-trichlorobenzene) in the several CMBRs run for each compound. One hundred milligrams of sediment was then added to each reactor and the bottles sealed with aluminum crimp seals lined with Tuff-Bond teflon discs. Controls containing no sorbent solids were run alongside the isotherm CMBRs to determine whether any significant losses of solute to the reactor surfaces were incurred. Generally the amount of solute mass recovered exceeded $95 \%$. The sealed CMBRs were sonicated to break up any large aggregates of sorbent solids and then placed on a rotary tumbler for a time sufficient to allow solution/sorbent equilibration.

Bottle-point CMBR rate studies were conducted to determine the time required to attain equilibrium in the isotherm tests. The same amount of sorbent was added to each of a series of CMBRs. A $10-\mu \mathrm{g} \mathrm{I}^{-1}$ working solution of the target compound was then added to each reactor and the reactors were agitated on a rotary tumbler. At regular intervals a different CMBR was removed from the tumbler, and its solids separated from the liquid phase by passing the suspension through two $25-\mathrm{mm}$ glass fiber filters housed in a stainless steel holder (Anspec Inc.). Controls indicated that losses to the filters were $<5 \%$. The aliquots were then extracted with hexane and assayed for residual concentration of solute. It was observed from these rate studies that no significant change in solute concentration occurred after approx. $100-120 \mathrm{~h}$. An equilibration period of $120 \mathrm{~h}$ was thus selected for use in all isotherm tests.

Following equilibration, the isotherm CMBRs were centrifuged at $2000 \mathrm{rpm}$ for $1 \mathrm{~h}$ to separate the solid and liquid phases. A portion of the supernatant was pipetted out and extracted with pesticide-grade hexane for approx. $30 \mathrm{~min}$. The phases were allowed to separate, and the hexane was assayed on a Hewlett-Packard 5880 gas chromatograph equipped with a ${ }^{63} \mathrm{Ni}$ electron capture detector and a glass column packed with a stationary phase suited to the particular compound being analysed. The instrument was operated isothermally using a methane-argon mobile phase.

All isotherm experiments involving volatile organic compounds were performed using a no-headspace technique Either $2 \mathrm{~g}$ of soil or $0.04 \mathrm{~g}$ of sediment were placed into each of a number of $40-\mathrm{ml}$ centrifuge tubes. A known amount of target solute in an acetone carrier was injected into $100 \mathrm{ml}$ of water, and this working solution was quickly added to the centrifuge tubes. The bottles were then sealed with Teflon-lined screw caps and gently tapped to remove any air bubbles. The caps were removed, more solution was added to completely fill the vials, and the reactors were then sealed again. The remainder of the experimental procedure was carried out in the manner prescribed for the isotherm tests with nonvolatile compounds.

\section{High performance liquid chromatography}

The high performance liquid chromatography instrument used in this study was comprised of a solvent delivery system (Waters Assoc. M45 pump), a variable wavelength detector (Waters Assoc. Lambda Max detector), a rotary injector fitted with a $20 \mu 1$ sample loop (Rheodyne Co.), and a $5 \mu-\mathrm{C} 18$ reverse phase Econosphere cartridge column system (Alltech Assoc.). The HPLC was interfaced to a Hewlett-Packard 3390A integrator, which recorded solute retention times.

This solutes examined in this phase of the study included those investigated in the sorption isotherm experiments plus several other pesticides and polynuclear aromatic hydrocarbons (PAHs). The target compounds were made up in acetone, which also served as the internal standard, and eluted isocratically with a methanol:water mix $(80: 20 \mathrm{v} / \mathrm{v})$. The mobile phase comprised of HPLC grade Millipore Norganic water and HPLC grade methanol (Fisher $\mathrm{Co}$.), and was degassed prior to use to eliminate potential interferences. Solute samples were run in duplicate at a flow rate of $1 \mathrm{ml} \mathrm{min}^{-1}$, and at a wavelength of either 254 or $221 \mathrm{~nm}$, depending upon the particular compound being analyzed.

\section{RESULTS AND DISCUSSION}

Partitioning of hydrophobic compounds onto soils and sediments

Representative sediment isotherms for $2,5,2^{\prime}-\mathbf{P C B}$, chlordane, and 1,2,4-trichlorobenzene are illustrated 


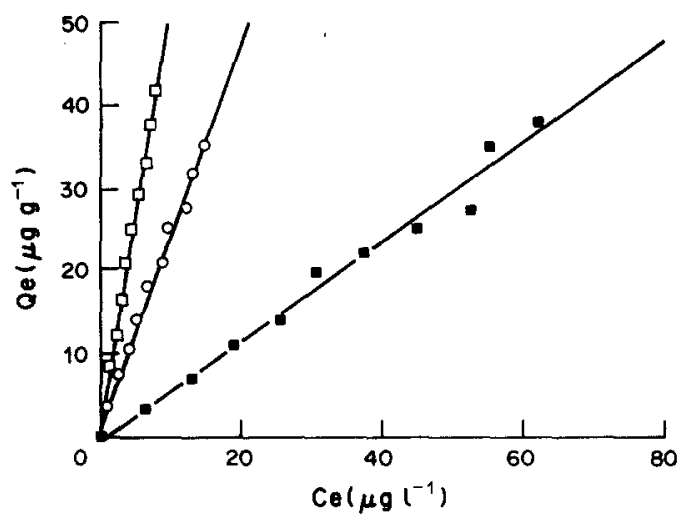

Fig. 1. Sorption of three HOCs on OSH sediment. Chlordane; $\bigcirc, 2,5,2$-PCB; $\mathbf{D}, 1,2,4-\mathrm{TCB}$

in Fig. 1. These are typical of the results obtained for both solids studied. The isotherm data for the seven target compounds was fitted to both the linearpartitioning and the Freundlich isotherm models. Data which exhibits truly linear trends should yield $n$ coefficient values approaching unity. The partition coefficients, Freundlich parameters and respective correlation coefficients resulting from the fitting of these two models to the experimental data are presented in Table 1. Judging from the correlation coefficients, both models appear to fit the data quite well. The majority of the slope values $(n)$ for the fitted Freundlich isotherms were reasonably close to unity, falling in a range from 0.85 to 1.07 . For purposes of comparing sorption isotherm data with HPRPLC data, the isotherm trends were sufficiently close to linear to allow approximate characterization in terms of the linear partition model.

The results of the sorption rate study described earlier indicated that the time required to attain equilibrium can be significant. It has long been assumed and often observed that sorption of hydrophobic organic compounds onto natural solids occurs within a rather small frame (hours). The rate profile illustrated in Fig. 2 for sorption of 2,5,2'-PCB onto OSH lacustrine sediments is representative of rate profiles generated for all systems studied in this work. It is readily apparent from Fig. 2 that a significant

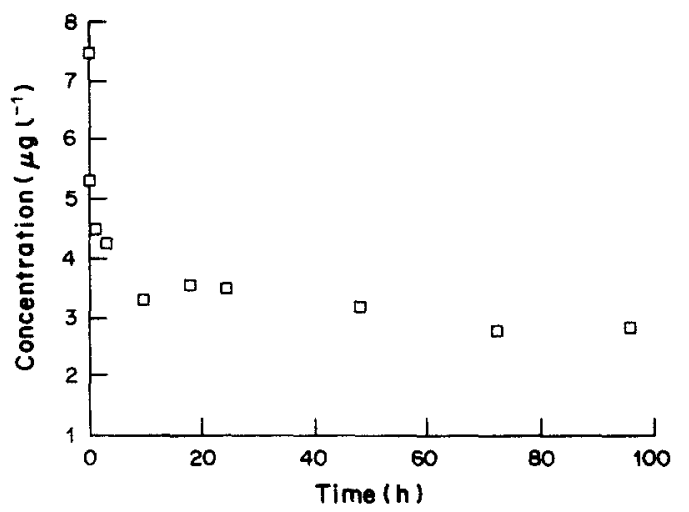

Fig. 2. 2,5,2'-PCB bottle point rate study. $\square, 2,5,2^{\prime}$-PCB.

uptake of target compound occurs quickly, but that sorption subsequently continues slowly over an extended period. Miller and Weber (1986) observed similar behavior for sorption of lindane onto several different types of soil. Karickhoff (1980, 1984) suggested that such sorptive processes can be described by a two-component model involving: (1) a rapid component in which the solute quickly sorbs onto a readily available fraction of the sorbent; and (2) a slower component in which solute sorbs at the less accessible remaining sites. Miller and Weber $(1984,1986)$ suggested an alternative model predicated on a dual-resistance mass transfer concept, and demonstrated an increased resistance to mass transfer and thus lower rates of sorption as solute hydrophobicity increased. Wu and Gschwend (1986) employed a simple, single resistance diffusion model to fit sorption data, and also speculated that the rate of diffusion of a solute is dependent upon its relative hydrophobicity. The slow sorption process observed in the current work and by others may extend over periods of days or even weeks. Experimental partition coefficients and predictive models based on assumptions of rapid kinetics may therefore not sufficiently represent actual sorption conditions for hydrophobic compounds. As noted earlier, equilibrium isotherms determined in this work were allowed to run for $120 \mathrm{~h}$, after which

Table 1. Linear partition coefficients, Freundlich parameters and correlation coefficients for sorption of target compounds onto a lacustrine sediment and a sandy soil

\begin{tabular}{|c|c|c|c|c|c|}
\hline Compound & $K_{p}$ & $r$ & $\log K_{\mathrm{F}}$ & $n$ & $r$ \\
\hline \multicolumn{6}{|c|}{ On OSH lacustrine sediments } \\
\hline $2,2^{\prime}, 4,5-\mathrm{PCB}$ & 6193 & 0.994 & 0.90 & 0.87 & 0.996 \\
\hline $2,5,2^{\prime}-\mathrm{PCB}$ & 2295 & 0.996 & 0.51 & 0.88 & 0.997 \\
\hline $4,4^{\prime}-\mathrm{PCB}$ & 2668 & 0.988 & 0.52 & 0.92 & 0.992 \\
\hline Chlordane* & 5216 & 0.999 & 0.71 & 1.00 & 0.997 \\
\hline $1,2,4-\mathrm{TCB} \dagger$ & 587 & 0.996 & 0.14 & 0.94 & 0.997 \\
\hline \multicolumn{6}{|c|}{ On Michawye soil $\left(\mathrm{cm} \mathrm{g}^{-1}\right)$} \\
\hline CCL4 & 0.542 & 0.996 & -3.08 & 0.96 & 0.988 \\
\hline $1,2,4-\mathrm{TCB}^{*}$ & 12.45 & 0.998 & -1.71 & 0.94 & 0.997 \\
\hline $2,5,2^{\prime} \cdot \mathrm{PCB}$ & 37.70 & 0.994 & -1.21 & 0.85 & 0.994 \\
\hline Chlordane & 182.7 & 0.990 & -0.78 & 1.07 & 0.991 \\
\hline
\end{tabular}

* cis isomer.

+Trichlorobenzene. 
Table 2. Logarithm of normalized carbon partition coefficients and retention times for the target compounds

\begin{tabular}{lcc}
\hline Compound & $\log \left(K_{\mathrm{oc}}\right)$ & $\log \left(t_{\mathrm{c}}\right)$ \\
\hline 2,2',4,5-PCB(OSH) & 5.64 & 1.22 \\
2,5,'-PCB(OSH) & 5.21 & 1.06 \\
4,4'-PCB(OSH) & 5.27 & 1.13 \\
Chlordane(OSH) & 5.57 & 1.25 \\
Trichlorobenzene(OSH) & 4.61 & 0.89 \\
CCL4 (Michawye) & 2.62 & 0.39 \\
Lindane (Michawye) & 3.42 & 0.67 \\
1,2,4-TCB (Michawye) & 3.98 & 0.89 \\
2,5,2'-PCB (Michawye) & 4.46 & 1.06 \\
Chlordane & 5.15 & 1.25 \\
\hline
\end{tabular}

*Lindane $K_{\mathrm{p}}$ value from Miller and Weber (1986). The sorption isotherms were done in a similar fashion, and $K_{\alpha c}$ determined from the organic fraction of the sorbent.

no noticeable further decrease in solution phase concentration occurred. It cannot necessarily be assumed, however, that the experimental systems were at true thermodynamic equilibrium after $120 \mathrm{~h}$. The condition is therefore characterized as an "apparent" equilibrium.

Correlations between partition coefficients and HPRPLC retention times

Experimentally determined partition coefficients and corresponding HPRPLC corrected retention times, $t_{c}$, for the six target compounds and the two sorbents studied are listed in Table 2. The $K_{\mathrm{oc}}$ value for sorption of lindane by Michawye soil was taken from the work of Miller and Weber (1986), in which the isotherm was measured under conditions similar to those described in this paper. Corrected retention times (the difference between a solute and a relatively unretained internal standard retention time) are used in lieu of capacity factors because unretained tracers commonly utilized to determine the reverse phase column void volumes yield different retention times (Krstulovic et al., 1982; Chin et al., 1986). Acetone was selected as the internal standard to which all target compounds were referenced; it was found to be relatively unretained, innocuous to the system, and easily detected.

The soil $K_{\mathrm{oc}}$ values for chlordane, trichlorobenzene, and $2,5,2^{\prime}$-PCB differ from their respective $\mathrm{OSH}$ $K_{o c}$ values by approximately one-half order of magnitude. This may be due in part to the low organic carbon content of the soil relative to the sediment. Karickhoff et al. (1979) observed that the normalization of partition coefficients to sorbent organic matter is applicable only to solids having organic carbon contents greater than $0.1 \%$. They noted that $\log \left(K_{o c}\right)$ values for sandy materials $\left(f_{o c}<0.01\right)$ were consistently lower than those determined for organic-rich sediments.

The so-called "solids effect" may also account in part for the lower effective soil $K_{o c}$ value because the solids levels employed in experiments with the soils were approximately two orders of magnitude greater than those employed in the tests on the lacustrine sediments. Several investigators (O'Conner and Connolly, 1980; Voice et al., 1983) have observed that the apparent partition coefficient for a hydrophobic compound can sometimes decrease with increasing solids to water ratios. Several explanations have been proposed (Curl and Keolian, 1983; Gschwend and $\mathrm{Wu}, 1985$; Voice and Weber, 1985), and there is reasonably general agreement that natural solids may release organic constituents (dissolved and/or colloidal) which may potentially interact with a target compound to change its sorption characteristics. Gschwend and Wu (1985) and Voice et al. (1983) observed that the amounts of dissolved organic carbon and colloidal material released were dependent upon the amount of solids present. It is apparent in any event that the normalizing of soil/sediment partition coefficients to organic carbon content of the solid phase may be limited in validity by system-specific factors.

Correlations relating $K_{\mathrm{oc}}$ and $t_{\mathrm{oc}}$ for the solutes listed in Table 2 are plotted in Fig. 3. The linear regression analyses of the $\mathrm{OSH}$ and Michawye $K_{o c}-t_{\mathrm{c}}$ data give relationships of the form

$$
\begin{aligned}
\log \left(K_{\mathrm{oc}}\right)_{\mathrm{OSH}} & =2.78 \log \left(t_{\mathrm{c}}\right)+2.17 ; \\
r & =0.982, \quad n=5
\end{aligned}
$$

and

$$
\begin{aligned}
\log \left(K_{\mathrm{oc}}\right)_{\mathrm{MICH} .} & =2.88 \log \left(t_{\mathrm{c}}\right)+1.47 ; \\
r & =0.998, \quad n=5 .
\end{aligned}
$$

It is evident from the coefficients of determination that a good correlation exists between $\log \left(K_{\text {oc }}\right)$ and HPRPLC retention times for the systems tested. This is significant because any relationship associating the sorptive behavior of an uncharged compound to its chromatographic behavior in a reverse phase column allows $K_{\mathrm{oc}}$ values to be estimated quickly from a measurement of the target compound's retention time. In addition, the ratio of the coefficient (slope of the generated lines) for the two equations is approximately unity, which seems to indicate that the sorptive process is dominated in both systems by the solute aqueous phase activity coefficient. The different $y$-intercept values seem to indicate that

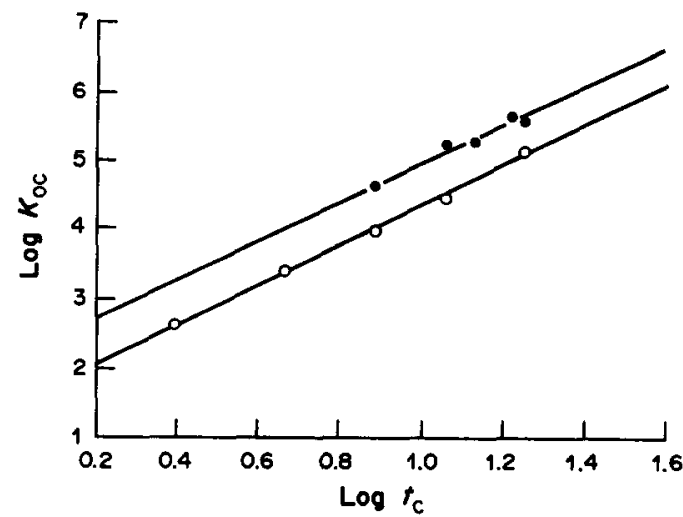

Fig. 3. Correlation between $K_{o c}$ and $t_{c}$. O, OSH; $O$, Michawye. 
Table 3. Correlation between $K_{\alpha c}$, the octanol/water partition coefficients, and the mole fraction aqueous solubilities of the target substances

\begin{tabular}{lcccc}
\hline Compound & $\log \left(K_{\text {oc }}\right)$ & $T_{\mathrm{m}}\left({ }^{\circ} \mathrm{C}\right)$ & $\log \left(K_{\text {ow }}\right)$ & $-\log \left(X_{i}^{w}\right)$ \\
\hline 2,2',4,5-PCB(OSH) & 5.64 & $47 \S$ & $6.67^{*}$ & $8.57 \S$ \\
2,5, 2'-PCB(OSH) & 5.21 & $44^{*}$ & $6.00^{*}$ & $7.83^{*}$ \\
4,4'-PCB(OSH) & 5.27 & $149^{*}$ & $5.32^{*}$ & $7.7^{*}$ \\
1,2,4-TCB(OSH) & 4.61 & $17^{*}$ & $3.98 \uparrow$ & $5.46^{*}$ \\
Chlordane (OSH) & 5.57 & $107 \dagger$ & $6.00 \|$ & $7.78 \dagger$ \\
CCLA (Michawy) & 2.62 & $-23 \dagger$ & $2.64 \dagger$ & $4.03 \dagger$ \\
Lindane (Michawye) & 3.42 & $112.9 \dagger$ & $3.72 \dagger$ & $5.46 \ddagger$ \\
1,2,4-TCB (Michawye) & 3.98 & 17 & 3.98 & 5.46 \\
2,5,2'-PCB (Michawye) & 4.46 & 44 & 6.00 & 7.83 \\
Chlordane Michawye & 5.15 & 107 & 6.00 & 7.78 \\
\hline
\end{tabular}

*From Yalkowsky et al. (1983); †Callahan (1979); †Karickhoff (1981); §Miller et al. (1985); \Chin et al. (1986); \|MacKay (1982).

the solute interaction with the sorbent-associated organic phase is different for the two solids studied. This observation reinforces the hypothesis that sorption onto natural solids is highly system-specific, and that the normalization of partition coefficients to sorbent target cannot be applied universally. It should also be stressed that retention times for any given set of organic solutes vary with different HPRPLC instruments, because they are dependent upon column design; i.e. column dimensions and the composition, particle size and configuration of the stationary phase. The correlation of $t_{\mathrm{c}}$ to $K_{\mathrm{oc}}$ values for a system of interest must first be established for a particular instrument and column. Reverse phase columns deteriorate over time, and retention times must be periodically checked to determine potential changes in resolution and precision. In addition, retention times and partition coefficients are sensitive to temperature fluctuations. An increase in temperature will tend to decrease both the retention time of an organic solute, and possibly $K_{\mathrm{oc}}$. Thus it is important to maintain a constant temperature for the duration of the experiment that is reflective of natural waters.

$K_{\mathrm{oc}}$ values for the solutes studied were also correlated to corresponding octanol/water partition coefficients and mole fraction solubilities. Values for the latter are presented in Table 3 . The regression plot

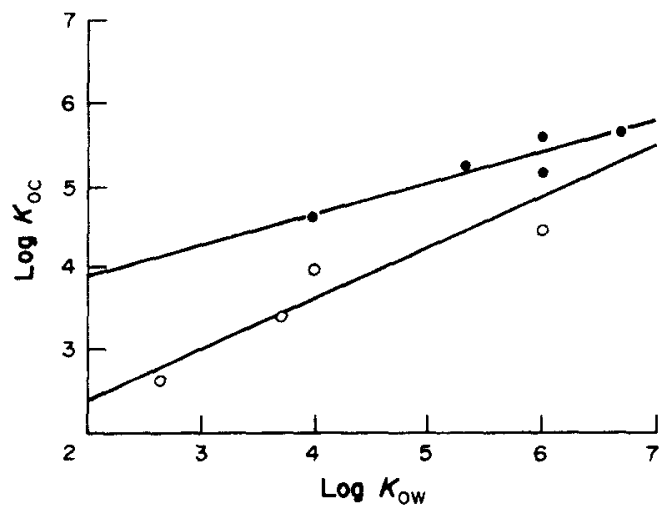

Fig. 4. Correlation between $K_{\mathrm{ow}}$ asnd $K_{\mathrm{oc}}$. 0 , OSH; 0 , Michawye. of $K_{\mathrm{oc}}$ and $K_{\mathrm{ow}}$ values is given in Fig. 4. Regression analyses of the OSH and Michawye data yields

$$
\begin{aligned}
\log \left(K_{\mathrm{oc}}\right)_{\mathrm{OSH}} & =0.374 \log \left(K_{\mathrm{ow}}\right)+3.16 ; \\
r & =0.94, \quad n=5
\end{aligned}
$$

and

$$
\begin{aligned}
\log \left(K_{\mathrm{oc}}\right)_{\mathrm{MICH}} & =0.615 \log \left(K_{\mathrm{ow}}\right)+1.18 ; \\
r & =0.94, \quad n=5 .
\end{aligned}
$$

The slope and intercept values for both regression analysis agree reasonably well with those determined by Kenaga and Goring (1980), who measured a $K_{\mathrm{oc}}-K_{\text {ow }}$ relationship of the form

$$
\log \left(K_{\mathrm{oc}}\right)=0.544 \log \left(K_{\mathrm{ow}}\right)+1.377
$$

for soils and sediments.

Although it is evident that a reasonably good relationship exists between $K_{o c}$ and the octanol/water partition coefficient, the correlation is less satisfactory than that between $K_{o c}$ and HPRPLC corrected retention time, $t_{\mathrm{c}}$. This is true for both the soil and sediment studied. The fact that the observed correlation between $\log \left(K_{o c}\right)$ and $\log \left(K_{\text {ow }}\right)$ is poorer could be due, in part, to the effect of mutual saturation and/or, in part, to inaccurate literature values for $K_{\mathrm{oc}}$, which vary by as much as an order of magnitude for certain compounds. The effects of water in octanol and octanol in water on the partition characteristics of an uncharged hydrophobic compound has been observed to alter the solute activity coefficient in both phases (Chiou et al., 1983; Miller et al., 1985; Chin et al., 1986). Further, it cannot be assumed that the behavior of a solute with respect to octanol will be similar to its association with heterogeneous organic geopolymer phases associated with solid sorbents, while solute interactions with the reverse phase resin may be more realistic. The octanol/water partitioning coefficient can provide a "first approximation" of the partitioning tendencies for a particular organic solute, while the HPRPLC model can be used to more accurately described and quantify solute sorption in soil/sediment systems.

A plot of $\log \left(K_{\mathrm{oc}}\right)$ and mole fraction solubility, $X_{i}^{\mathrm{W}}$ is given in Fig. 5. Linear regression fitting of the OSH 


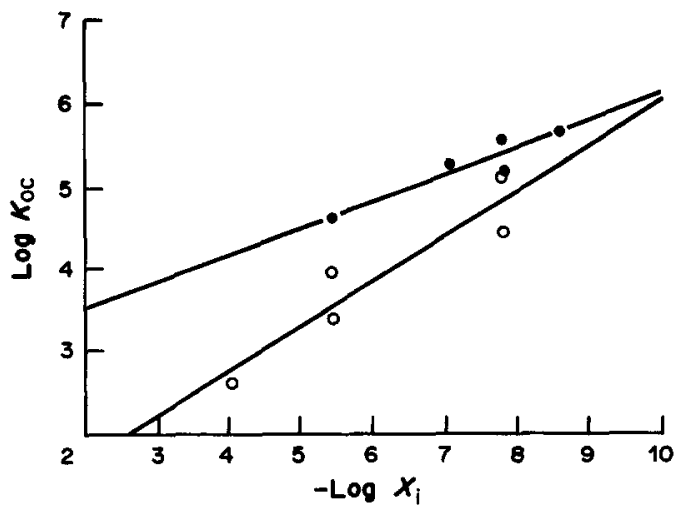

Fig. 5. Correlation between $K_{\mathrm{oc}}$ and solubility., $\mathrm{OSH}$; $\mathrm{O}$, Michawye.

and Michawye data yields

$$
\begin{aligned}
\log \left(K_{\mathrm{oc}}\right)_{\mathrm{OSH}}= & \frac{-\Delta S_{\mathrm{f}}\left(T_{\mathrm{m}}-T\right)}{2.3 R T} \\
& -0.324 \log \left(X_{i}^{\mathrm{w}}\right)+2.88 \\
r= & 0.94, \quad n=5
\end{aligned}
$$

and

$$
\begin{aligned}
\log \left(K_{\mathrm{oc}}\right)_{\mathrm{MICH} .}= & \frac{-\Delta S_{\mathrm{f}}\left(T_{\mathrm{m}}-T\right)}{2.3 R T} \\
& -0.547 \log \left(X_{i}^{\mathrm{w}}\right)+0.58 ; \\
r= & 0.93, n=5 .
\end{aligned}
$$

It is apparent that this correlation between $K_{o c}$ and aqueous solubility are less satisfactory than those between $K_{o c}$ and $t_{c}$, even when the activity of the solute is corrected to its supercooled liquid phase. This is probably due in large measure to the wide discrepancies commonly observed in reported solubility values. Experimental determination of aqueous solubility can be exceedingly difficult for a number of reasons, including compound instability, the presence of impurities, and slow rates of approach to equilibrium. These problems are especially prominent for very hydrophobic organic substances (Chin et al., 1986). Reported values for the solubility of $p, p^{\prime}$-DDT, for example, range over two orders of magnitude (Hakfenschied and Tomlinson, 1981). The accuracy of a $K_{\infty}$ estimate based on aqueous solubility is highly dependent upon the accuracy of the measured value, as well as on that of other parameters required in performing the estimate (e.g. the solute melting point, and entropy of fusion if the compounds are nonrigid) (Karickhoff, 1981).

Carbon normalized partition coefficient estimates derived from equations (8) and (9) are presented in Table 4. The predicted $\log \left(K_{\mathrm{oc}} \mathrm{s}\right)$ are compared in this table to experimental values from similar natural sorbent/water systems. The agreement between HPRPLC-estimated and observed $\log \left(K_{o c}\right)$ values is good, with an average absolute deviation of $0.18 \mathrm{log}$ units for the high-solids system and $0.25 \log$ units for the low solids system. Larger deviations do occur for the low-solids data because the determination of linear distribution coefficients is analytically more difficult and subject to greater error. It appears from both the measured and predicted $K_{\mathrm{oc}}$ values that sorption behavior is specific to the type and amount of solid present. The HPRPLC approach thus requires calibration of solute retention time to a particular system if reliable estimates are to be expected.

\section{CONCLUSIONS}

Investigation of the sorption and HPRPLC retention characteristics of several hydrophobic organic solutes with respect to different solids indicates that HPRPLC corrected retention time provides a reasonable basis for estimation of linear partitioning behavior. A HPRPLC retention time- $\log \left(\boldsymbol{K}_{o c}\right)$ model has been derived using a thermodynamic approach, and experimental data obtained to evaluate the coefficients of that model. Absolute average deviations between $K_{\mathrm{oc}}$ values estimated by the model are well below a half order of magnitude. Correlations between $K_{o c}, K_{o w}$ and aqueous solubility have also been established, but these in general provide a poorer characterization of $K_{o c}$. The HPRPLC retention time technique has potential as a means for elucidating the sorptive properties of geochemically and environmentally significant substances, and, when coupled with a qualitative analytical instrument (e.g. mass spectroscopy), can also be used to quickly identify and determine the sorptive properties of diagentically altered organic compounds.

Acknowledgements-The authors thank Dr Brian J. Eadie of the Great Lakes Environmental Research Laboratory of the National Oceanographic and Atmospheric Administration and Dr John W. Farrington of the Woods Hole Oceanographic Institute for their helpful comments. Assistance in the data analysis was provided by Kevin Olmstead

Table 4. HPRPLC-estimated and observed $K_{o c}$ values

\begin{tabular}{lccccc}
\hline Compound & $\log \left(t_{c}\right)$ & $\log \left(K_{\mathrm{oc}}\right)_{\mathrm{p}}^{*}$ & $\log \left(K_{\mathrm{oc}}\right)_{\circ}$ & $\log \left(K_{\mathrm{oc}}\right)_{\mathrm{p}}^{\dagger}$ & $\log \left(K_{\mathrm{oc}}\right)_{\mathrm{o}}$ \\
\hline Phenanthrene & 0.95 & 4.81 & $5.10 \ddagger$ & 4.20 & $4.08 \S$ \\
Naphthalene & 0.62 & 3.89 & $3.52 \dagger^{\dagger}$ & 3.25 & $3.11 \|$ \\
Pyrene & 1.16 & 5.40 & $5.40 \ddagger$ & 4.81 & $4.88 \S$ \\
p.p-DDT & 1.30 & 5.78 & $6.25{ }^{\circ}$ & 5.21 & $5.14 \|$ \\
$p$-DCB & 0.63 & 3.92 & $4.04^{* *}$ & 3.28 & 2.78 \\
\hline
\end{tabular}

"Low solids to water ratio. †High solids to water ratio. $\$ B$. J. Eadie, unpublished data; \&Karickhoff et al. (1979) and Karickhoff (1981); \Pierce et al. (1974); \|Schwarzenbach and Westall (1981); **Measured for OSH for this paper; †+Voice and Weber (1985). 
of the University of Michigan Environmental and Water Resources Program. This publication is, in part a result of work sponsored by the Michigan Seagrant College Program, Project No. R/TS-29 under Grant No. NA86AA-0-SG043 from the Office of Seagrant, National Oceanic and Atmospheric Administration (NOAA), U.S. Department of Commerce, and funds from the State of Michigan. Additional aspects of the research were carried out as part of National Science Foundation project ECE8503903, Environmental Engineering Program. The U.S government is authorized to produce and distribute reprints for governmental purposes not withstanding any copyright notation appearing hereon.

\section{REFERENCES}

Arbuckle W. B. (1983) Estimating activity coefficients for use in calculating environmental parameters. Envir. Sci. Technol. 17, 537-542.

Bopp R. F., Simpson H. J., Olsen C. R., Trier R. M. and Kostyk N. (1982) Chlorinated hydrocarbons and radionuclide chronologies in sediments of the Hudson River and Estuary, New York. Envir. Sci. Technol. 16, 666-676.

Brown D. S. and Flagg E. W. (1981) Empirical prediction of organic pollutant sorption in natural sediments. $J$. envir. Qual. 10, 382-385.

Brownawell B. J. (1986) The role of colloidal organic matter in the marine geochemistry of PCBs. Ph.D. dissertation, Woods Hole Oceanographic Institute.

Brownawell B. J. and Farrington J. W. (1986) Biogeochemistry of PCBs in interstial waters of a coastal marine sediment. Geochim. cosmochim. Acta 50, 157-169.

Burkhard L. P., Kuehl D. W. and Vieth G. (1985) Evaluation of reverse phase liquid chromatography/mass spectrometry for estimation of $n$-octanol/water partition coefficients for organic chemicals. Chemosphere 14, 1551-1560.

Callahan (Ed.) (1979) Water Related Environmental Fate of 129 Priority Pollutants, EPA440/4-79-029a/029b.

Chin Y. P., Weber W. J. Jr and Voice T. C. (1986) Determination of partition coefficients and aqueous solubilities by reverse phase chromatography-II. Evaluation of partitioning and solubility models. Wat. Res. 20 , 1443-1451.

Chiou C. T., Porter P. E. and Schmedding D. W. (1983) Partitioning equilibria of nonionic compounds between soil organic matter and water. Envir. Sci. Technol. 17, 227-231.

Chiou C. T., Schmedding D. W. and Manes M. (1982) Partitioning of organic compounds in octanol-water systems. Envir. Sci. Technol. 16, 4-10.

Chiou C. T., Freed V. H., Schmedding D. W. and Kohnert R. L. (1979) A physical concept of soil-water equilibria for nonionic compounds. Science 213, 683-684

Curl R. L. and Keolian G. A. (1984) Implicit-adsorbate model for apparent anomalies with organic adsorption on natural adsorbents. Envir. Sci. Technol. 18, 916-922.

D'amboise M. and Hanai T. (1982) Hydrophobicity and retention in reverse phase liquid chromatography. J. Liquid Chromat. 229-244.

Eadsforth C. V. and Moser P. (1983) Assessment of reverse phase chromatographic methods for determining partition coefficients. Chemosphere 12, 1459-1475.

Fujita T., Iwasa J. and Hansch C. (1964) A new substituent coefficient, $\pi$, derived from partition coefficients. $J . A m$. chem. Soc. 86, 5175-5180.

Gschwend P. M. and $W_{u}$ S. (1985) On the constancy of sediment-water partition coefficients of hydrophobic organic pollutants. Envir. Sci. Technol. 19, 90-96.

Hakfenschied T. L. and Tomlinson E. (1981) Estimation of aqueous solubilities of organic nonelectrolytes using liquid chromatography data. J. Chromat. 218, 409-425.
Haque R. and Schmedding D. W. (1975) A method of measuring the water solubility of hydrophobic chemicals: solubility of five polychlorinated biphenyls. Bull. envir contam. Toxic. 14, 13-18.

Karickhoff S. W. (1980) Sorption kinetics of hydrophobic pollutants in sediments. Contaminants and Sediments (Edited by Baker R.), Vol. 2, pp. 193-205. Ann Arbor Science, Ann Arbor, Mich.

Karickhoff S. W. (1981) Semi-empirical estimation of sorption of hydrophobic pollutants on natural sediments and soils. Chemosphere 10, 833-846.

Karickhoff S. W. (1984) Pollutant sorption in aquatic systems. J. Hydraul. Engng Am. Soc. civ. Engrs 10, $707-735$

Karickhoff S. W. and Brown D. S. (1979) Determination of octanol/water distribution coefficients, water solubilities, and sediment/water partition coefficients for hydrophobic organic pollutants. EPA-600/4-79-032, Athens.

Karickhoff S. W., Brown D. S. and Scott T. A. (1979) Sorption of hydrophobic pollutants on natural sediments. Wat. Res. 13, 241-248.

Kenaga E. E. and Goring C. A. I. (1980) Relationship between water solubility, soil-sorption, octanol/water partitioning, and bioconcentration of chemicals in biota In Aquatic Toxicology (Edited by Eaton J. G., Parish P. R. and Hendricks A. C.), ASTM STP707, pp. 78-115.

Krstulovic A. M., Colin H. and Guichon G. (1982) Comparison of methods used for the determination of the void volume in reverse phase chromatography. Analyt. Chem. 54, 2438-2443.

Lambert S. M., Porter P. E. and Schieferstein H. (1965) Movement and sorption of chemicals applied to the soil. Weeds 13, 185 190.

Leo A. and Hansch C. (1979) Substituent Constant for Correlation Analysis in Chemistry and Biology. Wiley. New York.

Mackay D. (1982) Correlation of bioconcentration factors Envir. Sci. Technol. 16, 274-278.

McCall J. M. (1975) Liquid-liquid partition coefficients by high performance liquid chromatography. J. med. Chem 18, 549-552.

McDuffie B. (1980) Estimation of octanol/water partition coefficients for organic pollutants using reverse phase high performance liquid chromatography. Chemosphere 10, 73-83.

Means J. C., Wood S. G., Hasset J. J. and Banwsart W. L. (1980) Sorption of polycyclic aromatic hydrocarbons by sediments and soils. Envir. Sci. Technol. 14, 1524-1528.

Means J. C., Wood S. G., Hassett J. J. and Banwart W. L. (1982) Sorption of amino- and carboxyl-substituted polynuclear aromatic hydrocarbons by sediments and soils. Envir. Sci. Technol. 16, 93-98.

Miller C. T. and Weber W. J. Jr (1984) Modeling organic contaminant partitioning in groundwater systems. Groundwater 22, 584-592.

Miller C. T. and Weber W. J, Jr (1986) Sorption of hydrophobic organic pollutants in saturated soil systems Contam. Hydrol. 1, 243-261.

Miller M. M., Wasik S. P., Huang G., Shiu W. and MacKay D. (1985) Relationship between octanol/water partition coefficient and aqueous solubility. Envir. Sci. Technol. 19, $522-529$

O'Conner D. J. and Connolly J. P. (1980) The effect of concentration of adsorbing solids on the partition coefficient. Wat. Res. 14, 1517-1523.

Pierce R. H., Olney C. E. and Felback G. T. (1974) pp'-DDT adsorption to suspended particulate matter in seawater. Geochim. cosmochim. Acta 38, 1061-1073.

Rapaport R. A. and Eisenreich S. J. (1984) Chromatographic determination of octanol-water partition coefficients $\left(K_{\mathrm{ow}}\right)$ for 58 polychlorinated biphenyl congeners. Envir. Sci. Technol. 18, 163-170.

Rekker R. F. (1977) The Hydrophobic Fragment Constant 
Its Derivitization and Application. A Means of Characterizing Membrane Systems. Elsevier, New York.

Schwarzenbach R. P. and Westall J. (1981) Transport of nonpolar organic compounds from surface water to groundwater. Laboratory sorption studies. Envir. Sci. Technol. 11, 1360-1367.

Sorrell R. K., Dressman R. C. and McFarren E. F. (1977) High pressure liquid chromotography for the measurement of polynuclear aromatic hydrocarbons in water. Presented at the Water Quality Technology Conference, Kansas City, MO.

Sutton C. and Calder J. A. (1974) Solubility of higher molecular weight n-paraffins in distilled water and seawater. Envir. Sci. Technol. 8, 654657.

Tsonopoulos C. and Prausnitz J. M. (1971) Activity coefficients of aromatic solutes in dilute aqueous solution. Ind. Engng Chem. Fund. 10, 593-599.

Vieth G. D., Austin N. M. and Morris R. T. (1979) A rapid method for estimating $\log \mathrm{P}$ for organic chemicals. Water. Res. 13, 43-47.

Voice T. C. and Weber W. J. Jr (1983) Sorption of hydrophobic compounds by sediments soils and suspended solids - I. Theory and Background. Wat. Res. 17, 1433-1441.

Voice T. C. and Weber W. J. Jr (1985) Solid effects in liquid/solid partitioning. Envir. Sci. Technol. 19, 789-796.

Voice T. C., Rice C. P. and Weber W. J. Jr (1983) Effect of solids concentration on the sorptive partitioning of hydrophobic pollutants in aquatic systems. Envir. Sci. Technol. 17, 513-517.

Wakeham S. G. and Farrington J. W. (1980) Hydrocarbons in contemporary aquatic sediments. In Contaminants and Sediments (Edited by Baker R.), Vol. 1, pp. 3-33. Ann Arbor Science, Ann Arbor, Mich.

Wakeham S. G., Schaffner C. and Giger W. (1979) Diagenetic polycyclic aromatic hydrocarbons in recent sediments: structural information obtained by high performance liquid chromatography. Adv. Organic Geochem. 12, 353-362.

Weber W. J. Jr, Chin Y. P. and Rice C. P. (1986) Determination of partition coefficients and aqueous solubilities by reverse phase chromatography-I. Theory and background. Wat. Res. 20, 1433-1442.

Weber W. J. Jr, Voice T. C., Pirbazari M., Hunt G. E. and Ulanoff D. M. (1983) Sorption of hydrophobic compounds by sediments, soils, and suspended solids-II. Sorbent evaluation studies. Wat. Res. 10, 1443-1452.

Wu S. and Gschwsend P. M. (1986) Sorption kinetics of hydrophobic compounds to natural sediments and soils. Envir. Sci. Technol. 20, 717-724.

Yalkowsky S. H. and Valvani S. C. (1980) Solubility and partitioning I: solubility of nonelectrolytes in water. J. Pharm. Sci. 69, 912-922.

Yalkowsky S. H., Valvani S. C. and MacKay D. (1983) Estimation of the aqueous solubility of some aromatic compounds. Residue Rev. 85, 43-55. 